Received: 20 November 2017

Accepted: 2 March 2018

Published online: 15 March 2018
CIENTIFIC REP

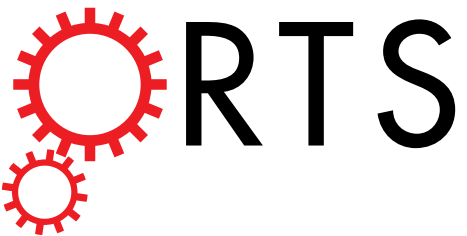

\section{OPEN Value of Preoperative Modified Body Mass Index in Predicting Postoperative 1-Year Mortality}

Tak Kyu Oh', ${ }^{1,2}$ Jaebong Lee ${ }^{3}$, Jung-Won Hwang ${ }^{2}$, Sang-Hwan Do ${ }^{2}$, Young-Tae Jeon ${ }^{2}$, Jin Hee Kim², Kooknam Kim² \& In-Ae Song ${ }^{1,2}$

Serum albumin and conventional BMI (CBMI) are commonly used indices of malnutrition status. Because CBMI does not reflect fluid accumulation, modified body mass index (mBMI, serum albumin $\times$ CBMI) is a more accurate measure of malnutrition status. This study investigated the association between preoperative $\mathrm{mBMI}$ and postoperative 1-year mortality, in comparison with serum albumin and CBMI. Medical records of 80,969 adult patients who underwent surgical procedures in a tertiary care hospital between 1 January, 2011 and 31 December, 2015 were retrospectively reviewed. Postoperative 1 -year mortality increased with reduction in CBMI, $\mathrm{mBMI}$, and albumin separately $(P<0.001)$. When considering interaction between $\mathrm{CBMI}$ and albumin, albumin was the only significant factor of postoperative 1 -year mortality [odds ratio: $0.377,95 \%$ confidence interval $(0.245-0.579), P<0.001$ ], while CBMI or interaction (CBMI * albumin) was not significant $(P>0.05)$. Adjusted area under the curve (AUC) was highest ( 0.885$)$ in the overall model (CBMI + albumin + CBMI * albumin); adjusted AUC between $\mathrm{mBMI}$ and albumin did not differ significantly $(P=0.558)$. Low albumin is the strongest independent predictor of postoperative 1-year mortality among the three variables (albumin, CBMI, $\mathrm{mBMI}$ ). Adding (BMI to albumin does not increase the validity of the AUC of albumin.

Malnutrition, a common complication of chronic or severe disease, is known to exacerbate disease prognoses ${ }^{1}$. Patients' malnutrition status may have adverse effects on their postoperative mortality or prognosis ${ }^{2}$. Therefore, preoperative malnutrition is an important clinical problem; conventional body mass index (cBMI) and serum albumin level are commonly used as indicators of malnutrition status ${ }^{3,4}$. Recent studies have reported that low preoperative $\mathrm{BMI}^{5,6}$ and low serum albumin level $\mathrm{l}^{7,8}$ exacerbate postoperative mortality and prognosis. This indicates the importance of preoperative nutritional status given the recent trend of increasing number of surgeries among elderly patients.

However, it is still not clear how accurately cBMI measures the nutritional status of patients ${ }^{10,11}$, because it has limitations in reflecting fluid balance, such as fluid accumulation or dehydration. In fact, such limitations of cBMI were suggested to affect patients undergoing liver transplantation, in which ascites is a common problem. A new approach suggested to overcome these limitations, is the use of the modified BMI $(\mathrm{mBMI})^{12,13}$. The mBMI considers serum albumin level and $\mathrm{cBMI}$ in combination, and thus is a relevant tool for patients who undergo liver transplantation ${ }^{10-13}$. However, there are no data on the effects of mBMI on the postoperative prognosis in the general population.

Therefore, we aimed to investigate the value of preoperative $\mathrm{mBMI}$ as a predictor of postoperative mortality, as compared to previously used predictors (cBMI and serum and albumin).

\section{Results}

A total of 82,452 adult patients underwent 104,660 cases of operations or surgical procedures at Seoul National University Bundang Hospital (SNUBH) between January 2011 and December 2015. Of these, 1,483 patients were excluded due to inaccurate or incomplete medical records; the remaining 80,969 patients were included in the

${ }^{1}$ Interdepartment of Critical Care Medicine, Seoul National University Bundang Hospital, 82, Gumi-ro 173 Beon-gil, Bundang-gu, Seongnam-si, Gyeonggi-do, 463-707, Korea. '2Department of Anesthesiology and Pain Medicine, Seoul National University Bundang Hospital, 82, Gumi-ro 173 Beon-gil, Bundang-gu, Seongnam-si, Gyeonggi-do, 463-707, Korea. ${ }^{3}$ Medical Research Collaborating Center, Seoul National University Bundang Hospital, 82, Gumi-ro 173 Beongil, Bundang-gu, Seongnam-si, Gyeonggi-do, 463-707, Korea. Correspondence and requests for materials should be addressed to I.-A.S. (email: songoficu@outlook.kr) 


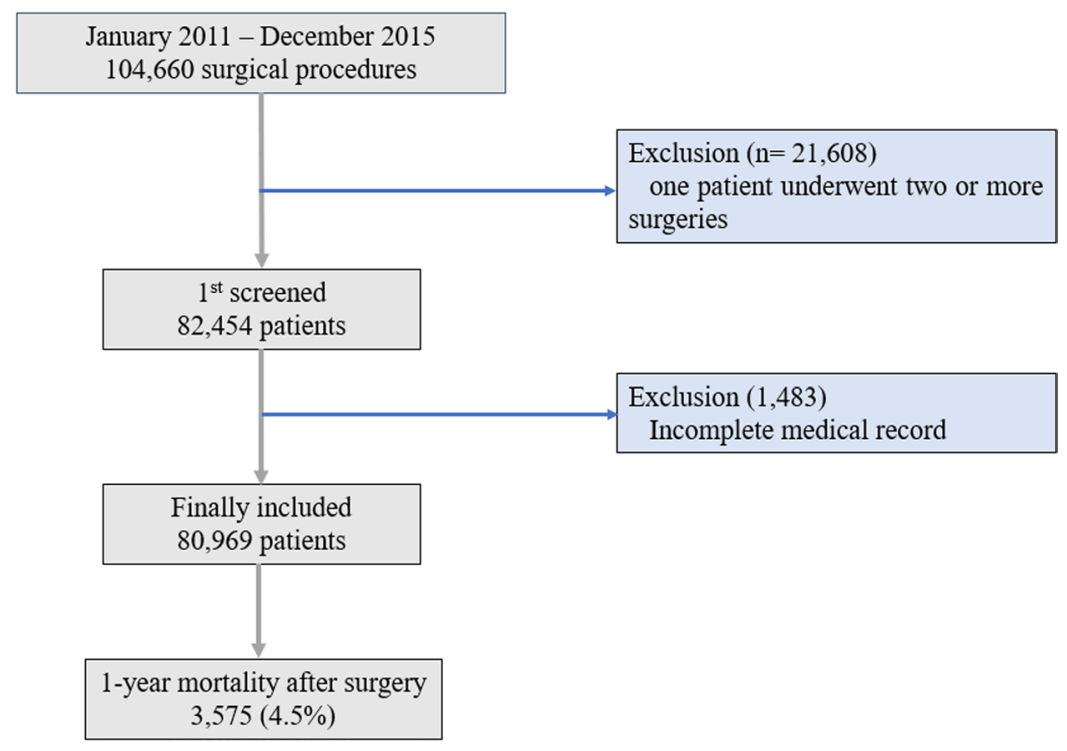

Figure 1. Flow chart for patient selection.

final analysis. Among the 80,969 patients, 3,575 (4.4\%) died within 1 year of the operation (Fig. 1). The demographic and clinical characteristics of the final analysis are presented in Table 1.

One-year mortality with respect to preoperative $\mathrm{cBMI}, \mathrm{mBMI}$, and albumin. The (log) ORs of postoperative 1-year mortality were plotted against changes in preoperative cBMI, mBMI, and albumin (Fig. 2A-C). Table 2 shows the univariate logistic regression analysis for postoperative 1-year mortality in all patients. Supplemental Table 1 shows the results of individual multivariate logistic regression model without considering the interaction between cBMI and albumin. Lower cBMI $(0.847,95 \%$ confidence interval [CI]: 0.836-0.858), mBMI (0.995, 95\% CI: 0.995-0.995), and albumin (0.230, 95\% CI: 0.214-0.247) were associated with increased postoperative 1-year mortality separately. However, in the multivariate model as shown in Table 3, when interaction among the three variables were considered, only albumin was significantly associated with postoperative 1 -year mortality [OR: $0.377,95 \%$ CI (0.245-0.579), $P<0.001$ ], whereas interaction (cBMI * albumin) and cBMI showed no significant association with postoperative 1-year mortality $(P>0.05)$.

Comparison of preoperative $\mathrm{CBMI}, \mathrm{mBMI}$, and albumin according to ROC curve. Figure $3 \mathrm{~A}$ presents the ROC curves that show the risk for postoperative 1-year mortality with respect to preoperative cBMI, $\mathrm{mBMI}$, and albumin. Figure $3 \mathrm{~B}$ presents the ROC curves that predict the risk for postoperative 1-year mortality with respect to the preoperative cBMI, $\mathrm{mBMI}$, and albumin after adjusting for the covariates (type of operation, gender, age, history of diabetes mellitus, hypertension, and ischaemic heart disease, ASA classification, history of general anesthesia, type of anesthesia, postoperative ICU admission). Prior to adjusting for the covariates, the AUC were $\mathrm{cBMI}$ Model $=0.659, \mathrm{mBMI}$ Model $=0.797$, albumin $\mathrm{Model}=0.811$, and overall Model $=0.820$ $(\mathrm{cBMI}+$ albumin $+\mathrm{cBMI} *$ albumin); Table 4 . After adjustment for the covariates, AUC was highest with the overall Model [cBMI + albumin + cBMI * albumin; 0.885, 95\% CI (0.878-0.891)], followed by mBMI [0.879, 95\% CI (0.873-0.886)], albumin [0.878, 95\% CI (0.872-0.885)], and cBMI [0.853, 95\% CI (0.846-0.860)]; Table 4. In addition, covariate adjusted AUC of mBMI was higher than $\mathrm{CBMI}(P<0.001)$, while covariate adjusted AUC of $\mathrm{mBMI}$ and albumin was not significantly different in DeLong's test $(P=0.558)$.

\section{Discussion}

Our study showed that low cBMI, mBMI, and albumin are risk factors of postoperative 1-year mortality; however, when considering the interaction between $\mathrm{CBMI}$ and albumin, albumin was the only significant factor of postoperative 1-year mortality. Furthermore, adding cBMI to albumin does not increase the validity of the AUC of albumin in predicting postoperative 1-year mortality. These findings are important and meaningful, because they were obtained from the general population of more than 80,000 patients over five years at a 1,360-bed tertiary care hospital. Furthermore, this is the first study that used mBMI in general population cohorts; mBMI has been previously used for liver transplantation patients.

The first thing to consider when interpreting our findings is the superiority between serum albumin and cBMI in representing patients' malnutrition status. A previous study reported that for patients on dialysis, albumin was an independent risk factor of operative mortality or morbidity, while $\mathrm{cBMI}$ was not ${ }^{3}$. In contrast, low cBMI was associated with mortality in patients undergoing cardiac surgery, while serum albumin was not ${ }^{14}$. Therefore, it is imperative to identify the index with a greater sensitivity for postoperative prognosis. We verified that albumin is a better index than $\mathrm{CBMI}$ in predicting postoperative prognosis. The reason for this superiority of albumin is that it reflects protein energy malnutrition unlike $c B M I$, which simply reflects the nutritional status of patients ${ }^{15}$. Low serum albumin is a measure of exacerbated immunity ${ }^{16}$ and a risk factor of increased postoperative infection ${ }^{17,18}$. Therefore, serum albumin is a better predictor of postoperative prognosis than cBMI. 


\begin{tabular}{|c|c|c|c|}
\hline Total $(\mathrm{n}=80,969)$ & Number (\%) & Mean & SD \\
\hline \multicolumn{4}{|l|}{ Operation } \\
\hline Cardiovascular surgery & $1,134(1.4 \%)$ & & \\
\hline Non-cardiovascular surgery & $79,835(98.6 \%)$ & & \\
\hline Male (\%) & $35,302(43.6 \%)$ & & \\
\hline Age (year) & & 54.5 & 16.2 \\
\hline cBMI $\left(\mathrm{kg} / \mathrm{m}^{2}\right)$ & & 24.1 & 3.6 \\
\hline \multicolumn{4}{|l|}{ Preoperative Laboratory Test Result } \\
\hline Hemoglobin $(\mathrm{g} / \mathrm{dL})$ & & 13.5 & 1.9 \\
\hline WBC (x1000/uL) & & 6.7 & 2.4 \\
\hline Platelet (x1000/uL) & & 246.3 & 71.0 \\
\hline Prothrombin time (INR) & & 1.1 & 0.1 \\
\hline Aspartate aminotransferase (IU/L) & & 24.6 & 104.0 \\
\hline Alanine aminotransferase (IU/L) & & 24.4 & 41.6 \\
\hline Albumin $(\mathrm{g} / \mathrm{dL})$ & & 4.3 & 0.5 \\
\hline Glucose (mg/dL) & & 109.0 & 35.9 \\
\hline aPTT (sec) & & 36.7 & 5.9 \\
\hline Blood urea nitrogen $(\mathrm{mg} / \mathrm{dL})$ & & 14.7 & 7.4 \\
\hline Serum creatinine $(\mathrm{mg} / \mathrm{dL})$ & & 0.9 & 0.8 \\
\hline Serum sodium $(\mathrm{mmol} / \mathrm{L})$ & & 140.4 & 2.8 \\
\hline Serum potassium $(\mathrm{mmol} / \mathrm{L})$ & & 4.2 & 0.4 \\
\hline \multicolumn{4}{|l|}{ Cormack grade } \\
\hline $\mathrm{I}, \mathrm{II}$ & $67,771(83.7 \%)$ & & \\
\hline III, IV & $13,198(16.3 \%)$ & & \\
\hline \multicolumn{4}{|l|}{ Preoperative Comorbidity } \\
\hline Diabetes mellitus & $9,635(11.9 \%)$ & & \\
\hline Hypertension & $20,404(25.2 \%)$ & & \\
\hline Ischemic heart disease & $4,534(5.6 \%)$ & & \\
\hline Neurologic disease & $3,400(4.2 \%)$ & & \\
\hline \multicolumn{4}{|l|}{ ASA classification } \\
\hline I & $36,921(45.6 \%)$ & & \\
\hline II & $39,350(48.6 \%)$ & & \\
\hline III & $4,534(5.6 \%)$ & & \\
\hline IV, V, VI & $162(0.2 \%)$ & & \\
\hline \multicolumn{4}{|l|}{ Type of anesthesia } \\
\hline History of general anesthesia & $31,578(39.0 \%)$ & & \\
\hline General anesthesia & $54,897(67.8 \%)$ & & \\
\hline Regional anesthesia & $10,607(13.1 \%)$ & & \\
\hline Monitored anesthesia care & $14,103(17.4 \%)$ & & \\
\hline Local anesthesia & $1,281(1.6 \%)$ & & \\
\hline Postoperative ICU admission & $3.7 \%$ & & \\
\hline
\end{tabular}

Table 1. Baseline characteristics of all patients. SD, standard deviation; $c B M I$, conventional body mass index; WBC, white blood cell; ASA, American Society of Anesthesiologists; aPTT, activated partial thromboplastin time; ICU, intensive care unit.

We showed that $\mathrm{mBMI}$ has an equal predictive power to serum albumin with postoperative 1-year mortality. In addition, when interactions between albumin and cBMI were considered, albumin was the only independent predictor of postoperative 1-year mortality. It means that serum albumin was the most important factor for predicting 1-year mortality rather than cBMI. Previous studies have hypothesised that $\mathrm{mBMI}$ would be superior to cBMI in predicting mortality because it also measures the effects of fluid accumulation; therefore, mBMI would be more important for patients with ascites or edema ${ }^{2,10}$. Similarly, our study showed that $\mathrm{mBMI}$ is a stronger predictor of postoperative mortality is than $\mathrm{CBMI}$ in postoperative patients. We hypothesised that mBMI would be better than $\mathrm{CBMI}$ and serum albumin because $\mathrm{mBMI}$ has the characteristics of cBMI and albumin used in predicting postoperative prognosis. However, our study showed that low mBMI was not a better indicator of postoperative 1-year mortality than serum albumin, and adding cBMI to albumin does not increase the validity of the AUC of albumin in predicting postoperative 1-year mortality. Furthermore, mBMI was also not an independent risk factor when considering the interactions among variables. Based on this, we suggest that $\mathrm{mBMI}$ is a more appropriate index than only cBMI for use in patients undergoing surgery. Moreover, using mBMI is not 

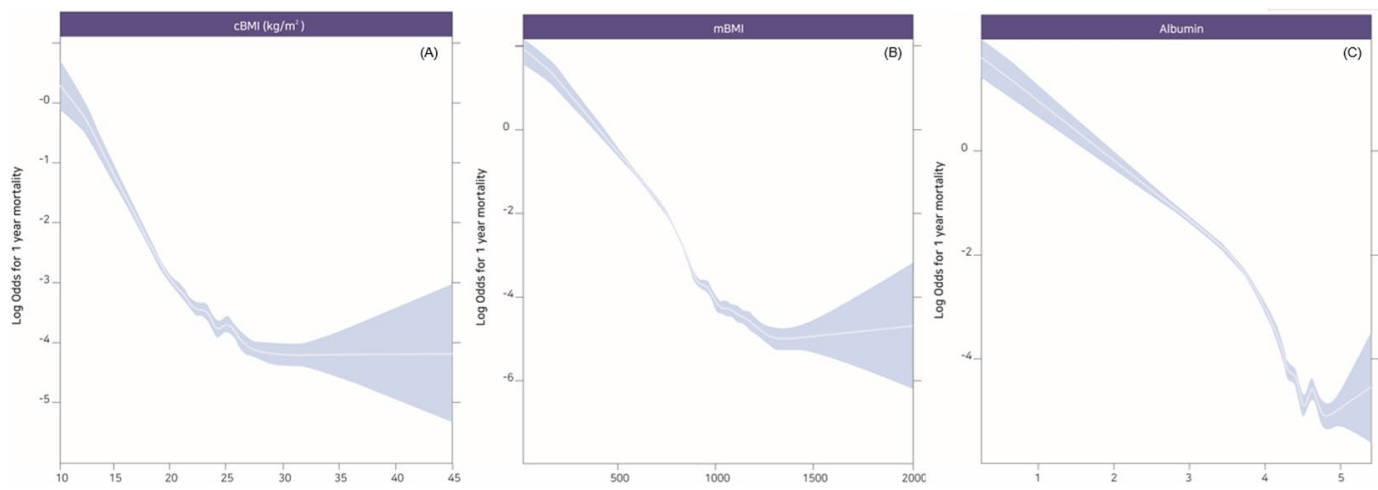

Figure 2. Log odds for postoperative 1-year mortality plotted against the changes of preoperative cBMI (A), mBMI (B), and albumin $(\mathbf{C})$.

recommended for predicting postoperative 1-year mortality rather than serum albumin; because serum albumin is the strongest index for predicting 1-year mortality after surgery.

In addition, there is an important consideration for using $\mathrm{mBMI}$; unlike albumin levels or cBMI, which are currently the standard indices, there are no clear classification criteria for the normal ranges of mBMI. Tanaka et al. classified mBMI into 6 groups $(<600,600-800,800-1000,1000-1200,1200-1400 \text {, and }>1400)^{11}$. Suhr et al. reported that mortality was high in the liver transplantation group with $\mathrm{mBMI}<600^{12}$. However, no other study has classified mBMI more appropriately and investigated its association with mortality. Since mBMI involves a wider range of measurements (600-1400) than albumin or cBMI, the OR of mBMI seems smaller than that of cBMI or albumin. Considering this wide range of $\mathrm{mBMI}$, further studies are warranted to identify appropriate classifications of $\mathrm{mBMI}$ for its clinical application.

Our study has a few limitations. First, the retrospective design may have led to selection or detection bias. However, this analysis based on all general surgical adult populations, included a large sample size of more than 80,000 .

Second, the generalizability of our findings is limited because we reviewed the medical records at a single center. Third, serum albumin levels were checked at different time points across patients, so there may be differences in the measurements according to the disease severity. Finally, we could not take into consideration the surgical techniques used and postoperative care during the study period of 5 years. Nevertheless, our study is meaningful in that it is the first study that compared the association of mBMI, albumin, and cBMI with 1-year mortality in general surgical patients.

In conclusion, our study showed that low albumin was the strongest and independent risk factor of postoperative 1-year mortality compared to $\mathrm{cBMI}$ or $\mathrm{mBMI}$. Adding cBMI to albumin does not increase the validity of the AUC of albumin in predicting postoperative 1-year mortality.

\section{Methods}

This study was a retrospective observational study and was approved by the Institutional Review Board at the SNUBH (Approval Number: B1705/395-106). The requirement for written informed consent was waived by the IRB, and this manuscript adheres to the applicable STROBE guidelines. The medical records of adult patients who were admitted to the SNUBH between January 2011 and December 2015 and underwent an elective or emergency surgical procedure were collected. Patients with inaccurate or incomplete medical records were excluded from the analysis. When one patient underwent two or more surgeries, only the medical record for the final surgery was included. The SNUBH is a 1,360-bed tertiary care academic hospital, where about 150 elective or emergency surgical operations are performed in 38 operating rooms on average every day. Furthermore, the hospital has been keeping and managing medical records since 2003 using an electronic medical system.

Definition of mBMI. We defined and calculated mBMI using the method suggested by a previous study ${ }^{10,11}$. The $\mathrm{mBMI}$ was calculated by multiplying $\mathrm{cBMI}\left(\mathrm{kg} / \mathrm{m}^{2}\right)$ with preoperative serum albumin level $(\mathrm{g} / \mathrm{L})$. We used heights and weights taken before the surgery or at the time of admission and serum albumin level determined at the day closest to the surgery and at least 4 weeks prior to the surgery.

Data Collection and outcome. The following medical records were collected for the study: gender, age (year), height $(\mathrm{cm})$, weight $(\mathrm{kg}), \mathrm{cBMI}\left(\mathrm{kg} / \mathrm{m}^{2}\right)$, preoperative blood laboratory test results [haemoglobin $(\mathrm{g} / \mathrm{dL})$, white blood cell count $(* 1000 / \mu \mathrm{L})$, platelet count $(* 1000 / \mu \mathrm{L})$, prothrombin time (INR), aspartate aminotransferase (IU/L), alanine aminotransferase (IU/L), glucose $(\mathrm{mg} / \mathrm{dL})$, activated partial thromboplastic time (sec), blood urea nitrogen $(\mathrm{mg} / \mathrm{dL})$, creatinine $(\mathrm{mg} / \mathrm{dL})$, sodium $(\mathrm{mmol} / \mathrm{L})$, potassium $(\mathrm{mmol} / \mathrm{L})]$, Cormack grade, [history of diabetes mellitus, hypertension, ischaemic heart disease, and neurologic disease], American society of anesthesiologists (ASA) classification, history of general anesthesia, type of operation and anesthesia, postoperative ICU admission, and death date.

Only preoperative blood laboratory test results obtained within 1 month before the surgery were used. The type of operation was classified into cardiovascular surgery and non-cardiovascular surgery. Cardiovascular surgery was defined as cardiac surgery or major vascular surgery involving cardiopulmonary bypass. All medical 


\begin{tabular}{|c|c|c|c|c|}
\hline Variable & $\begin{array}{l}\text { 1-year death } \\
(\mathbf{n}=3,575)\end{array}$ & $P$-value & Odds ratio $(95 \% \mathrm{CI})$ & $P$-value \\
\hline Operation & & $<0.001$ & & \\
\hline Non-cardiovascular surgery & $3,428(95.9 \%)$ & & Ref & \\
\hline Cardiovascular surgery & $147(4.1 \%)$ & & $3.14(2.56-3.86)$ & $<0.001$ \\
\hline Gender & & $<0.001$ & & \\
\hline Male & $2,252(63.0 \%)$ & & Ref & \\
\hline Female & $1,323(37.0 \%)$ & & $0.44(0.41-0.48)$ & $<0.001$ \\
\hline Age $(y r)$, mean $(S D)$ & $67.5(13.9)$ & $<0.001$ & $1.06(1.06-1.07)$ & $<0.001$ \\
\hline cBMI $\left(\mathrm{kg} / \mathrm{m}^{2}\right)$, mean $(\mathrm{SD})$ & $22.09(3.70)$ & $<0.001$ & $0.83(0.82-0.84)$ & $<0.001$ \\
\hline$<18.5$ & $575(16.1 \%)$ & & Ref & \\
\hline $18.5-24.9$ & $2,266(63.4 \%)$ & & $0.25(0.23-0.28)$ & $<0.001$ \\
\hline $25-29.9$ & $661(18.5 \%)$ & & $0.14(0.12-0.16)$ & $<0.001$ \\
\hline$\geq 30$ & $73(2.0 \%)$ & & $0.09(0.07-0.12)$ & $<0.001$ \\
\hline mBMI, mean $(\mathrm{SD})$ & $805.9(223.7)$ & $<0.001$ & $0.99(0.99-0.99)$ & $<0.001$ \\
\hline Albumin $(\mathrm{g} / \mathrm{dL})$, mean $(\mathrm{SD})$ & $3.6(0.7)$ & $<0.001$ & & \\
\hline$<2.5$ & $182(5.1 \%)$ & & Ref & \\
\hline $2.5-3.5$ & $1,312(36.7 \%)$ & & $0.31(0.25-0.39)$ & $<0.001$ \\
\hline$>3.5$ & $2,081(58.2 \%)$ & & $0.03(0.03-0.04)$ & $<0.001$ \\
\hline Diabetes mellitus & $21.5 \%$ & $<0.001$ & $2.09(1.90-2.30)$ & $<0.001$ \\
\hline Hypertension & $33.3 \%$ & $<0.001$ & $1.51(1.39-1.64)$ & $<0.001$ \\
\hline Ischemic heart disease & $14.3 \%$ & $<0.001$ & $2.96(2.65-3.31)$ & $<0.001$ \\
\hline Neurologic disease & $10.0 \%$ & $<0.001$ & $2.65(2.33-3.02)$ & $<0.001$ \\
\hline \multicolumn{5}{|l|}{ ASA classification } \\
\hline I & $382(10.7 \%)$ & & Ref & \\
\hline II & $2,066(57.8 \%)$ & & $5.23(4.61-5.94)$ & $<0.001$ \\
\hline III & $1,047(29.3 \%)$ & & $26.23(23.43-30.94)$ & $<0.001$ \\
\hline IV & $58(1.9 \%)$ & & 72.76 (50.77-104.28) & $<0.001$ \\
\hline $\mathrm{V}$ & $12(0.3 \%)$ & & $31.83(8.94-113.40)$ & $<0.001$ \\
\hline VI & $10(0.3 \%)$ & & $79.58(25.88-244.72)$ & $<0.001$ \\
\hline History of general anesthesia & $2,045(57.2 \%)$ & $<0.001$ & $2.15(1.99-2.32)$ & $<0.001$ \\
\hline Type of anesthesia & & $<0.001$ & & \\
\hline General anesthesia & $2,313(64.7 \%)$ & & Ref & \\
\hline Regional anesthesia & $311(8.7 \%)$ & & $0.69(0.60-0.79)$ & $<0.001$ \\
\hline Monitored anesthesia care & $944(26.4 \%)$ & & $1.47(1.35-1.61)$ & $<0.001$ \\
\hline Local anesthesia & $8(0.2 \%)$ & & $8.99(3.31-24.40)$ & $<0.001$ \\
\hline Postoperative ICU admission & $626(17.5 \%)$ & $<0.001$ & $6.38(5.73-7.10)$ & $<0.001$ \\
\hline
\end{tabular}

Table 2. Univariate logistic regression analysis for 1-year mortality in all patients. SD, standard deviation; cBMI, conventional body mass index; mBMI, modified body mass index; ASA, American Society of Anesthesiologists; ICU, intensive care unit.

records were collected by a medical record technician of the SNUBH's medical informatics team, who was blinded from the aims of this study; the main researchers were also blinded from the data until the final statistical outcomes were available. Moreover, the accurate dates of death of all patients (as of July 1,2017) were obtained with approval from the Ministry of the Interior and Safety in Korea.

The primary outcome evaluated was the value of preoperative $\mathrm{mBMI}$ as a prognostic factor for postoperative 1-year mortality. Therefore, we compared mBMI with cBMI and albumin levels.

Statistical Methods. The baseline characteristics of all patients are presented as percentage (\%) or mean and standard deviation. Continuous variables were analysed using the $t$-test and categorical variables were analysed using the chi-square test to evaluate the association of each variable with 1-year survival or death. The risk for postoperative 1-year mortality with respect to the three variables (cBMI, mBMI, and albumin) was analysed using restricted cubic spline, and the odds ratios (ORs) for postoperative 1-year mortality for each variable were computed using univariate and multivariate logistic regression analysis. We performed univariate logistic regression analysis to determine factors associated with postoperative 1-year mortality individually. We then selected covariates significant at $P<0.05$ in the univariate logistic model to be included in the multivariate logistic regression analysis. At multivariate logistic regression analysis, we built two models considering the interaction among cBMI, mBMI, and albumin. Finally, the power of each of the three variables (cBMI, mBMI, and albumin) for explaining postoperative 1-year mortality was computed using the receiver operating characteristic (ROC) curve; areas under the curve (AUC) were compared and tested using the DeLong's test. Additionally, we used ROC 


\begin{tabular}{|c|c|c|}
\hline \multirow[b]{2}{*}{ Variable } & \multirow{2}{*}{\begin{tabular}{|l|} 
Multivariate model \\
Odds Ratio $(\mathbf{9 5} \% \mathrm{CI})$ \\
\end{tabular}} & \multirow[b]{2}{*}{$P$-value } \\
\hline & & \\
\hline \multicolumn{3}{|l|}{ Type of operation } \\
\hline Non-cardiovascular surgery & Ref & \\
\hline Cardiovascular surgery & $0.674(0.526-0.857)$ & 0.002 \\
\hline \multicolumn{3}{|l|}{ Gender } \\
\hline Male & Ref & \\
\hline Female & $0.547(0.50-0.60)$ & $<0.001$ \\
\hline Age (year) & $1.03(1.03-1.04)$ & $<0.001$ \\
\hline Diabetes mellitus & $1.03(0.92-1.15)$ & 0.621 \\
\hline Hypertension & $0.71(0.64-0.78)$ & $<0.001$ \\
\hline Ischemic Heart Disease & $0.86(0.74-0.99)$ & 0.035 \\
\hline Neurologic Disease & $0.97(0.83-1.14)$ & 0.738 \\
\hline \multicolumn{3}{|l|}{ ASA class } \\
\hline I & Ref & \\
\hline II & $2.83(2.44-3.29)$ & $<0.001$ \\
\hline III & $5.58(4.66-6.70)$ & $<0.001$ \\
\hline $\mathrm{IV}, \mathrm{V}, \mathrm{VI}$ & $11.37(7.43-17.26)$ & $<0.001$ \\
\hline \multirow[t]{2}{*}{ History of general anesthesia } & $\operatorname{Ref}(\mathrm{No})$ & \\
\hline & $1.228(1.17-1.40)$ & $<0.001$ \\
\hline \multicolumn{3}{|l|}{ Type of anesthesia } \\
\hline General anesthesia & Ref & \\
\hline Regional anesthesia & $0.53(0.45-0.61)$ & $<0.001$ \\
\hline Monitored anesthesia care & $1.09(0.98-1.22)$ & 0.100 \\
\hline Local anesthesia & $2.80(0.70-10.10)$ & 0.131 \\
\hline \multirow[t]{2}{*}{ Postoperative ICU admission } & Ref (No) & \\
\hline & $1.64(1.42-1.90)$ & $<0.001$ \\
\hline $\mathrm{cBMI}\left(\mathrm{kg} / \mathrm{m}^{2}\right)$ & $0.94(0.88-1.01)$ & 0.087 \\
\hline Interaction (cBMI * Albumin) & $0.98(0.97-1.00)$ & 0.076 \\
\hline Albumin & $0.38(0.25-0.58)$ & $<0.001$ \\
\hline
\end{tabular}

Table 3. Multivariate logistic regression analysis for 1-year mortality regarding three preoperative variables (cBMI, mBMI, and albumin). cBMI, conventional body mass index; mBMI, modified body mass index; IHD, ischemic heart disease; NUD, neurologic disease; ASA, American Society of Anesthesiologists; ICU, intensive care unit.
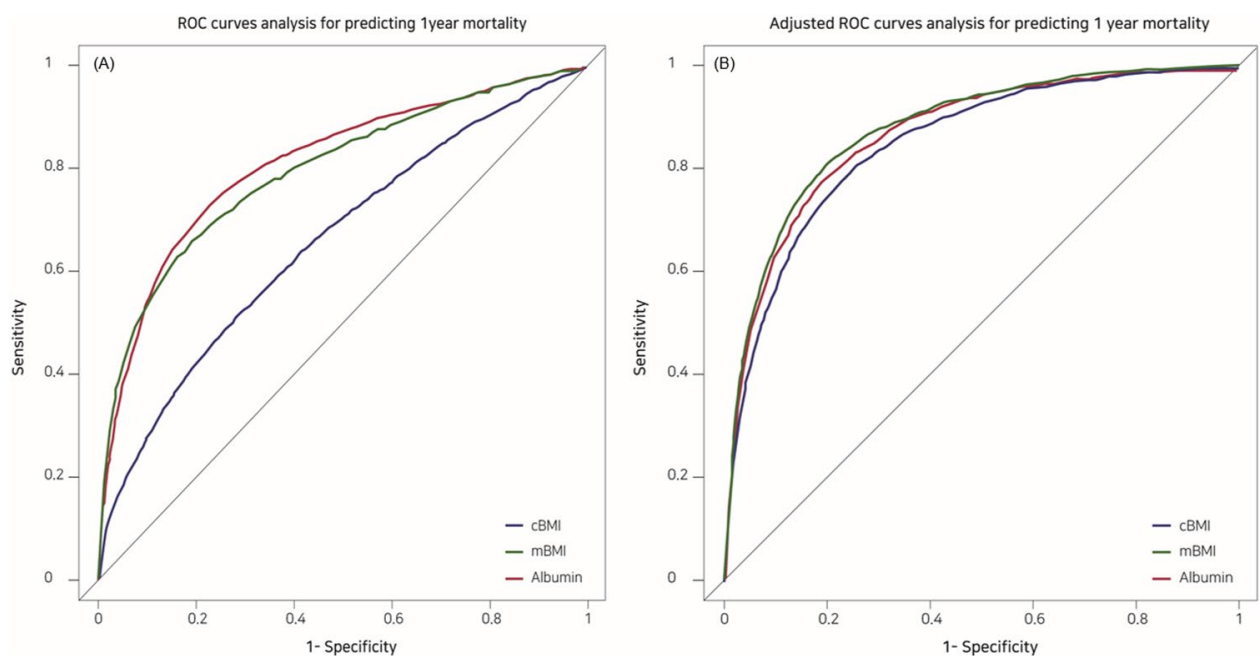

Figure 3. ROC curves (A) and covariate-adjusted ROC curves (B) showing the risk of postoperative 1-year mortality with respect to preoperative cBMI, mBMI, and albumin. 


\begin{tabular}{|l|l|l|l|l|}
\hline \multirow{2}{*}{ Variable } & \multirow{3}{*}{ 95\% Confidence Interval } \\
\cline { 4 - 5 } & & AUC & Lower Limit & Upper Limit \\
\hline \multirow{2}{*}{ cBMI Model } & Not adjusted & 0.659 & 0.648 & 0.670 \\
\cline { 2 - 5 } & Adjusted* $(1)$ & 0.853 & 0.846 & 0.860 \\
\hline \multirow{2}{*}{ mBMI Model } & Not adjusted & 0.797 & 0.787 & 0.807 \\
\cline { 2 - 5 } & Adjusted* $(2)$ & 0.879 & 0.873 & 0.886 \\
\hline \multirow{2}{*}{ Overamin Model } & Not adjusted & 0.811 & 0.802 & 0.820 \\
\cline { 2 - 5 } & Adjusted*(3) & 0.878 & 0.872 & 0.885 \\
\hline & Not adjusted & 0.820 & 0.811 & 0.829 \\
\cline { 2 - 5 } & Adjusted*(4) & 0.885 & 0.878 & 0.891 \\
\hline
\end{tabular}

Table 4. Comparison of three preoperative factors (cBMI, mBMI, and albumin) by ROC curve. *Model: adjusted covariates (type of operation, gender, age, history of diabetes mellitus, hypertension, ischemic heart disease, neurologic disease, American society of anesthesiologists classification, history of general anesthesia, type of anesthesia, postoperative intensive care unit admission). ** Overall Model: $\mathrm{cBMI}+$ albumin + interaction (cBMI * albumin). DeLong's test for two ROC curve, (1) vs (4): $\mathrm{Z}=16.673$, $P<0.001$, (2) vs (4): $Z=8.753, P<0.001$, (3) vs (4): $Z=6.670, P<0.001$, (2) vs (3): $Z=0.586, P=0.558$.

curves, after adjusting for the covariates that affected postoperative 1-year mortality, to compute adjusted AUC for each variable. The covariates adjusted for in the AUC were selected from the univariate logistic regression analysis based on the criterion of $P<0.05$.

The power of the sample size of our study was verified by the PASS 15 program. From the area under the curve (AUC) of 0.8 , to detect the difference of 0.05 of the AUC from the event rate of $4.4 \%, 15,000$ patients were adequate to yield $80 \%$ power and an alpha error of 0.05 . Therefore, our study was determined to have sufficient power to detect a difference of 0.05 from an AUC of 0.8 in predicting 1-year mortality. Statistical analyses were performed using IBM SPSS (Version 23.0; IBM Corp., Armonk, NY, USA) and R (Version 3.3.2 with R packages; http://www.R-project.org) software, with statistical significance set at $P<0.05$.

Data availability. The datasets generated during the current study are available from the corresponding author on reasonable request.

\section{References}

1. Norman, K., Pichard, C., Lochs, H. \& Pirlich, M. Prognostic impact of disease-related malnutrition. Clin Nutr 27, 5-15, https://doi. org/10.1016/j.clnu.2007.10.007 (2008).

2. Pikul, J., Sharpe, M. D., Lowndes, R. \& Ghent, C. N. Degree of preoperative malnutrition is predictive of postoperative morbidity and mortality in liver transplant recipients. Transplantation 57, 469-472 (1994).

3. Chan, M., Kelly, J., Batterham, M. \& Tapsell, L. Malnutrition (subjective global assessment) scores and serum albumin levels, but not body mass index values, at initiation of dialysis are independent predictors of mortality: a 10-year clinical cohort study. J Ren Nutr 22, 547-557, https://doi.org/10.1053/j.jrn.2011.11.002 (2012).

4. Hendifar, A. et al. Influence of Body Mass Index and Albumin on Perioperative Morbidity and Clinical Outcomes in Resected Pancreatic Adenocarcinoma. PLoS One 11, e0152172, https://doi.org/10.1371/journal.pone.0152172 (2016).

5. Matsuoka, K. et al. Significance of Body Mass Index for Postoperative Outcomes after Lung Cancer Surgery in Elderly Patients. World J Surg, https://doi.org/10.1007/s00268-017-4142-0 (2017).

6. Wu, N. et al. The prognostic influence of body mass index, resting energy expenditure and fasting blood glucose on postoperative patients with esophageal cancer. BMC Gastroenterol 16, 142, https://doi.org/10.1186/s12876-016-0549-6 (2016).

7. Brunaud, L. et al. Predictive factors for postoperative morbidity after laparoscopic adrenalectomy for pheochromocytoma: a multicenter retrospective analysis in 225 patients. Surg Endosc 30, 1051-1059, https://doi.org/10.1007/s00464-015-4294-7 (2016).

8. Caras, R. J., Lustik, M. B., Kern, S. Q., McMann, L. P. \& Sterbis, J. R. Preoperative Albumin Is Predictive of Early Postoperative Morbidity and Mortality in Common Urologic Oncologic Surgeries. Clin Genitourin Cancer 15, e255-e262, https://doi. org/10.1016/j.clgc.2016.09.008 (2017).

9. Puvanesarajah, V. et al. Poor Nutrition Status and Lumbar Spine Fusion Surgery in the Elderly: Readmissions, Complications, and Mortality. Spine (Phila Pa 1976) 42, 979-983, https://doi.org/10.1097/BRS.0000000000001969 (2017).

10. Suhr, O., Danielsson, A., Holmgren, G. \& Steen, L. Malnutrition and gastrointestinal dysfunction as prognostic factors for survival in familial amyloidotic polyneuropathy. J Intern Med 235, 479-485 (1994).

11. Tanaka, T., Renner, E. L., Selzner, N., Therapondos, G. \& Lilly, L. B. The impact of obesity as determined by modified body mass index on long-term outcome after liver transplantation: Canadian single-center experience. Transplant Proc 45, 2288-2294, https:// doi.org/10.1016/j.transproceed.2012.11.009 (2013).

12. Suhr, O. B., Ericzon, B. G. \& Friman, S. Long-term follow-up of survival of liver transplant recipients with familial amyloid polyneuropathy (Portuguese type). Liver Transpl 8, 787-794, https://doi.org/10.1053/jlts.2002.34386 (2002).

13. Yamamoto, S. et al. Liver transplantation for familial amyloidotic polyneuropathy (FAP): a single-center experience over 16 years. Am J Transplant 7, 2597-2604, https://doi.org/10.1111/j.1600-6143.2007.01969.x (2007).

14. Rapp-Kesek, D., Stahle, E. \& Karlsson, T. T. Body mass index and albumin in the preoperative evaluation of cardiac surgery patients. Clin Nutr 23, 1398-1404, https://doi.org/10.1016/j.clnu.2004.06.006 (2004).

15. Blackburn, G. L. \& Harvey, K. B. Prognostic strength of nutritional assessment. Prog Clin Biol Res 77, 689-697 (1981).

16. Gleeson, M. W. \& Dickson, R. C. Albumin gains immune boosting credibility. Clin Transl Gastroenterol 6, e86, https://doi. org/10.1038/ctg.2015.11 (2015).

17. Shi, H., Na, Q., Zhang, X. \& Jiang, X. Correlations between the levels of acute infection markers and serum albumin in elderly patients with hip fracture. Aging Clin Exp Res 29, 435-441, https://doi.org/10.1007/s40520-016-0585-7 (2017).

18. Yuwen, P. et al. Albumin and surgical site infection risk in orthopaedics: a meta-analysis. BMC Surg 17, 7, https://doi.org/10.1186/ s12893-016-0186-6 (2017). 


\section{Author Contributions}

Tak Kyu Oh contributed to the study design, and drafted the first manuscript; Jaebong Lee analysed the data. JungWon Hwang, Sang-Hwan Do, Jin Hee Kim, Young-Tae Jeon and Kooknam Kim contributed to the acquisition of data and provided critical revision of the manuscript. In-Ae Song contributed to the study design and provided critical revision of the manuscript. All authors read and approved the final manuscript.

\section{Additional Information}

Supplementary information accompanies this paper at https://doi.org/10.1038/s41598-018-22886-6.

Competing Interests: The authors declare no competing interests.

Publisher's note: Springer Nature remains neutral with regard to jurisdictional claims in published maps and institutional affiliations.

(c) (i) Open Access This article is licensed under a Creative Commons Attribution 4.0 International License, which permits use, sharing, adaptation, distribution and reproduction in any medium or format, as long as you give appropriate credit to the original author(s) and the source, provide a link to the Creative Commons license, and indicate if changes were made. The images or other third party material in this article are included in the article's Creative Commons license, unless indicated otherwise in a credit line to the material. If material is not included in the article's Creative Commons license and your intended use is not permitted by statutory regulation or exceeds the permitted use, you will need to obtain permission directly from the copyright holder. To view a copy of this license, visit http://creativecommons.org/licenses/by/4.0/.

(c) The Author(s) 2018 